\title{
Gene Expression Profiling of Human Adipocyte Responses to Insulin and IGF-I Signalling
}

\author{
Shaukat Mahmood ${ }^{1}$, Rehannah Borup ${ }^{2}$, Hans Tornqvist ${ }^{3}$, Kenneth J. O'Byrne ${ }^{4}$, Pierre De Meyts ${ }^{1}$ and \\ Steven G. Gray ${ }^{*}, 1,4$
}

${ }^{I}$ Hagedorn Research Institute, Receptor Systems Biology Laboratory, Novo Nordisk A/S, Niels Steensens Vej 6, DK2820 Gentofte, Denmark

${ }^{2}$ Rigshospitalet, Center of Diagnostic Investigation, Department of Clinical Biochemistry, Copenhagen, Denmark

${ }^{3}$ CVGI TA Clinical Early Development, Astra Zeneca R, Mölndal, 43183- Mölndal, Sweden

${ }^{4}$ Dept of Clinical Medicine, Translational Cancer Research Group, Institute of Molecular Medicine, Trinity Centre for Health Sciences, St James's Hospital, Dublin 8, Ireland

\begin{abstract}
A constant paradox for researchers working in the field of insulin and insulin-like growth factor-I (IGF-I), is to explain how these proteins despite binding to and activating highly homologous membrane receptors and triggering similar signalling pathways, yet exert distinct physiological roles in the control of metabolism and growth. Despite extensive studies, the molecular basis for the specificity between insulin and IGF-I signaling is still poorly understood. In an attempt to reveal the gene expression profiles regulated by their divergent functions, we used Affymetrix microarrays to monitor gene expression patterns that are regulated by either insulin or IGF-I. A fully differentiated human adipocyte line, derived from the adipose tissue of a child with Simpson-Golabi-Behmel Syndrome (SGBS), was stimulated with either insulin or IGF-I. Following stimulation of the differentiated adipocytes with insulin for $24 \mathrm{~h}$, we found 329 genes differentially regulated of which 215 were up-regulated and 114 were down-regulated. When SGBS adipocytes were stimulated with IGF-I, 103 genes were differentially expressed, of which 70 were up-regulated and 33 were down-regulated. Our results indicate that under the conditions used in the present study, insulin is a more potent regulator of gene expression in human adipocytes than IGF-I.
\end{abstract}

\section{INTRODUCTION}

The polypeptide hormones insulin and insulin-like growth factor-I (IGF-I) that belong to a structurally related superfamily are essential for growth, development, metabolism, and survival [1]. These hormones mediate their biological effects by binding to their cognate transmembrane receptors on the surface of the target cells. Although both the receptors and the ligands are structurally and functionally similar and target essentially the same intracellular signaling networks, the two receptors trigger distinct physiological responses [2,3]. The phenotype of homozygous knockout mice suggested that IGF-IR is primarily a growth promoter, while IR is a metabolic regulator [4]. When born, IR knockout mice are of normal size but subsequently develop severe hyperglycemia and hyperketonemia, while IGF-IR knockout mice exhibited severe abnormalities in growth and differentiation and die shortly after birth, primarily from lack of development of thoracic muscles [4].

Since both the IR and IGF-IR utilize essentially the same intracellular signaling networks, yet nevertheless trigger distinct physiological responses, it is crucial to delineate the differential gene responses elicited by these ligands to gain

*Address correspondence to this author at the Department of Clinical Medicine, Trinity Centre for Health Sciences, St. James's Hospital, Rm 2.09, James's Street, Dublin 8, Ireland; E-mail: sgray@stjames.ie an insight into how the specificity of ligand signaling is determined. Previously, attempts to molecularly dissect the effects of these signaling pathways on gene expression profiling studies have used mouse NIH-3T3 fibroblasts overexpressing either the IR or IGF-IR [5] or 3T3-L1 cells expressing receptor chimeras with identical extracellular domains fused to the intracellular domain of the IR or IGF-IR [6]. Recently, the gene expression profile induced by insulin and IGF-I has been studied in more relevant target cells like primary human myoblasts and myotubes, where IGF-I was found to be a more potent regulator than insulin [7]. Also, Jensen et al. compared the gene expression profile induced by insulin and an insulin mimetic peptide in rat L6 myoblasts transfected with the insulin receptor A-isoform [8].

In the present work, we chose to gain a global insight on gene expression patterns differentially regulated by insulin and IGF-I by using another major insulin target, adipocytes. We chose to use a human preadipocyte cell line, derived from the adipose tissue of a child with Simpson-GolabiBehmel syndrome (SGBS), a rare X-linked recessive syndrome characterized by pre- and postnatal overgrowth, facial dysmorphic features, supernumerary nipples, and a variety of other inconstant malformations. This human cell line has the ability to proliferate for many generations without losing the ability to undergo differentiation into an adipocyte, and represents a useful tool for molecular studies of human fat cell biology $[9,10]$. Pre-adipocytes and adipocytes were 
stimulated with either insulin or IGF-1, and gene expression profiles were determined using Affymetrix HG-U133A microarrays. Following this, validation of several genes which showed differential expression were performed by real-time PCR. Finally, the online Ingenuity Pathways Analysis software was used to identify key biological pathways and functions associated with the genes identified by microarray analysis.

\section{MATERIALS AND METHODS}

\section{Cell Culture}

Human SGBS preadipocytes (kindly provided by Prof. Martin Wabitsch, Ulm University) were cultured to near confluency in DMEM (Dulbecco's modified Eagle's medium) supplemented with $10 \%$ FCS (fetal calf serum). To induce adipose differentiation cells were repeatedly washed with PBS buffer and cultured in serum- and albumin-free DMEM containing $10 \mathrm{nM}$ human insulin (Novo Nordisk), 1 $\mu \mathrm{M}$ corticol (Sigma), and $200 \mathrm{pM}$ triiodothyronine (Day 0). Differentiation was initiated by the addition of Rosiglitazone for 3 days and Dexametasone/MIX (methylisobutylxanthine) for 7 days. Differentiation proceeded until day 14 followed by an additional 2 days without insulin (Day 16). At this time point, nearly all preadipocytes had acquired an adipocyte phenotype filled with multiple large fat droplets.

Under experimental conditions, the SGBS preadipocytes and adipocytes were starved for 24 hours in serum-free media, and subsequently treated with either human insulin (100 $\mathrm{nM})$ or IGF-I (6.58 nM - $50 \mathrm{ng} / \mathrm{ml})$ for 24 hours.

\section{RNA Isolation and Sample Processing}

Total RNA was extracted from two different passages in triplicates from each passage, using Tri-Reagent according to the manufacturer's protocol (Invitrogen; Carlsbad, Ca). The RNA isolated was pooled and further purified using the RNeasy mini kit according to the manufacturers protocol (Qiagen). Samples were processed for microarray analysis using the protocols and kits provided/recommended by the manufacturer (Affymetrix). Duplicate Affymetrix HGU133A (Affymetrix Inc., Santa Clara, CA, USA) arrays from each pool were carried out for each treatment condition. In total 4 arrays were used for each treatment, representing two biological replicates and two technical. Following hybridization, fluorescence intensities were determined using an Affymetrix scanner 3000 .

\section{Data Analysis}

Data analysis of scanned images was performed using the program dChip, developed by $\mathrm{Li}$ and Wong and its perfect match (PM)-only, model-based expression index [11]. Briefly, the array files were normalized using the multi array invariant-set normalization method. dChip was used to calculate significance at $\mathrm{P}<0.05$ of the $\mathrm{PM}$ data from the mean and standard errors of the signal intensities. The standard errors attached to the MBEI values are array-specific measurement errors, and dChip obtained these values by comparing the probe response pattern in a particular array to the pattern seen in most arrays [11] (also see http://biosun1.harvard.edu/complab/dchip/ ).

Transcripts which were identified by using a $90 \%$ lower confidence bound of fold change, and up or down-regulation by 1.5 -fold or more and a $\mathrm{P}<0.05$, were further analyzed for functional ontology using Ingenuity Pathways Analysis.

\section{Ingenuity Pathways Analysis}

Ingenuity Pathways Analysis (IPA) software (http://www.ingenuity.com/) was used to identify key functions and pathways differentially regulated by the two ligands, insulin and IGF-I. The data set obtained from dChip, containing gene identifiers and their corresponding expression values (fold change) were uploaded into IPA as a tabdelimited text file to perform the analysis. Each gene was mapped to its corresponding gene object in the IPA knowledge base.

\section{Real-Time PCR}

To validate microarray data, we chose seven genes belonging to different classes of categories and performed realtime quantitative PCR (qPCR). cDNA for real-time qPCR was generated for each sample using the Transcriptor First Strand cDNA Synthesis Kit (Roche diagnostics) according to manufacturer's instructions. Briefly, $100 \mathrm{ng}$ of total RNA from the same pooled aliquot of RNA as used for the microarray analysis was reverse transcribed using random hexamer primers, and the provided reverse transcriptase in a total volume of $20 \mu \mathrm{l}$, cDNA was stored at $-20^{\circ} \mathrm{C}$ until further use.

Universal Probe Library probes (Roche), were used for real-time PCR assays, and were designed using Probefinder (www.probelibrary.com). Real-time PCR assays were run using FastStart Taqman Probe master ROX (Roche) on the ABI prism 7900 Sequence Detection system (PE Applied Biosystems). Absolute quantification using a standard curve was used and 18s RNA was used for normalization. The PCR thermal cycling conditions performed for all of the samples was as follows: 10 minutes at $95^{\circ} \mathrm{C}$; and 40 cycles for the melting $\left(95^{\circ} \mathrm{C}\right.$ for 15 seconds) and annealing/extension steps $\left(60^{\circ} \mathrm{C}\right.$ for 1 minute). At least two independent experiments were performed in duplicates for each gene and compared with the results obtained by microarray analysis.

\section{RT-PCR}

$1^{\text {st }}$ strand cDNA was generated from $1 \mu \mathrm{g}$ of total RNA with Superscript II according to the manufacturers instructions (Invitrogen). Markers of adipocyte differentiation were examined using the primers and PCR conditions for Glut4, PPAR $\gamma 2$, and Leptin as previously described [12]. Quantification of the RT-PCR results was obtained by scanning the gel images and importing the data into TINA (Raytest $\mathrm{GmBH}$ ) with Beta-Actin utilized as the internal control. The values for the gene under scrutiny were normalized to the internal control.

\section{RESULTS}

\section{Genes Differentially Regulated by Insulin and/ or IGF-I in Human SGBS Adipocytes}

In order to analyze global gene expression patterns differentially regulated by either insulin or IGF-I, differentiated human SGBS adipocytes were stimulated by either human insulin (100 nM) or IGF-I (6.58 nM - $50 \mathrm{ng} / \mathrm{ml})$ for 24 hours. To confirm differentiation, the expression of three markers 
of differentiation (PPAR, Leptin and GLUT-4) was examined by RT-PCR in the pre-adipocytes and following differentiation into adipocytes (Fig. 1). Our results clearly demonstrate the induction of these markers of adipocyte differentiation as the cells move from the pre-adipocyte to adipocyte status, in agreement with others [10]. Folllowing extraction of total RNA, biotinylated cRNA probes were generated from RNA extracted from non-stimulated controls and ligand-stimulated SGBS cells and hybridized to Affymetrix HG-U133A arrays, according to Affymetrix procedure, and the data analyzed by DNA-Chip Analyzer (dChip). Gene lists, were generated by comparing the samples in the two treatments with untreated controls. Using this procedure (Fig. 2), we identified in total 329 transcripts that were differentially expressed by greater than 1.5 fold in insulin treatment and 103 transcripts in IGF-I treatment. Comparison analyses between genes differentially regulated by insulin and IGF-I, identified 244 genes that were specifically regulated by insulin and 18 specifically by IGF-I, whereas 85 were regulated by both insulin and IGF-I. Clustering of the genes regulated by insulin and IGF-I, revealed that the greatest responses in the samples were obtained by insulin (Fig. 3). These results indicate that, in SGBS adipocytes, insulin is a more potent regulator of gene transcription than IGF-I.

\section{Patterns of Gene Expression and Functional Analysis}

Considering the number of genes differentially regulated in this experimental setup, it is obvious that we cannot discuss all one by one. Therefore, we carried out functional analysis by using the Ingenuity Pathways Analysis (IPA). Data sets, obtained from dChip containing gene identifiers, corresponding expression values and $\mathrm{p}$-values were uploaded into the IPA application and genes associated with biological functions that were most significant to the particular dataset were identified, and Fischer's exact test was used to calculate a p-value for each biological function to that data set.

A
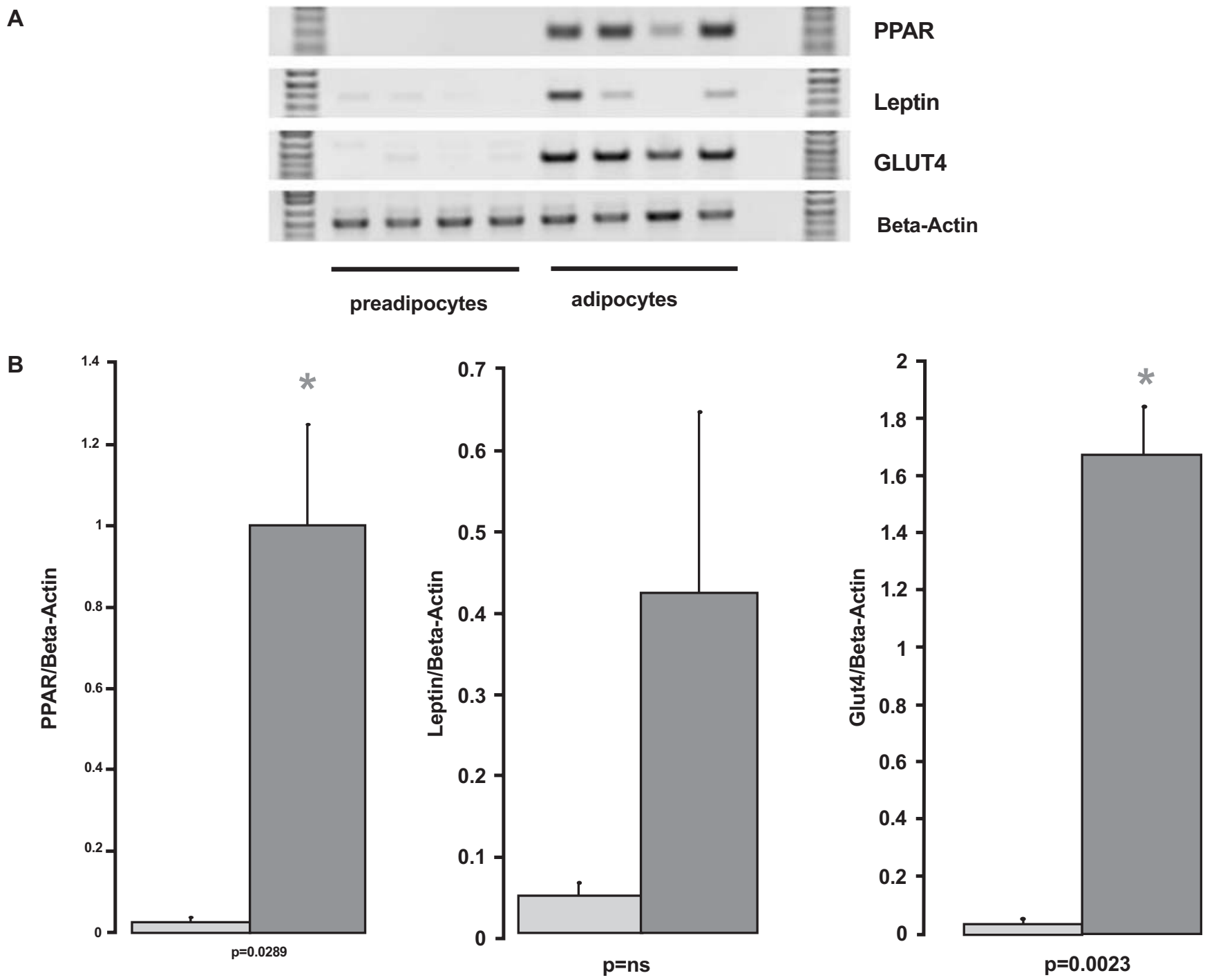

Fig. (1). Confirmation of SGBS adipocyte differentiation. (a) Expression of adipocyte differentiation markers were examined in a series of SGBS pre-adipocytes and adipocytes following differentiation. The results for four separate individual differentiations are shown confirming the elevated expression of these genes occurs in adipocytes following differentiation. (b) densitometric analysis of the changes in gene expression. The results are presented as the mean \pm standard error of the mean for each gene. Statistical significance (p-values) were calculated using a Students T-Test. ns = not significant. 


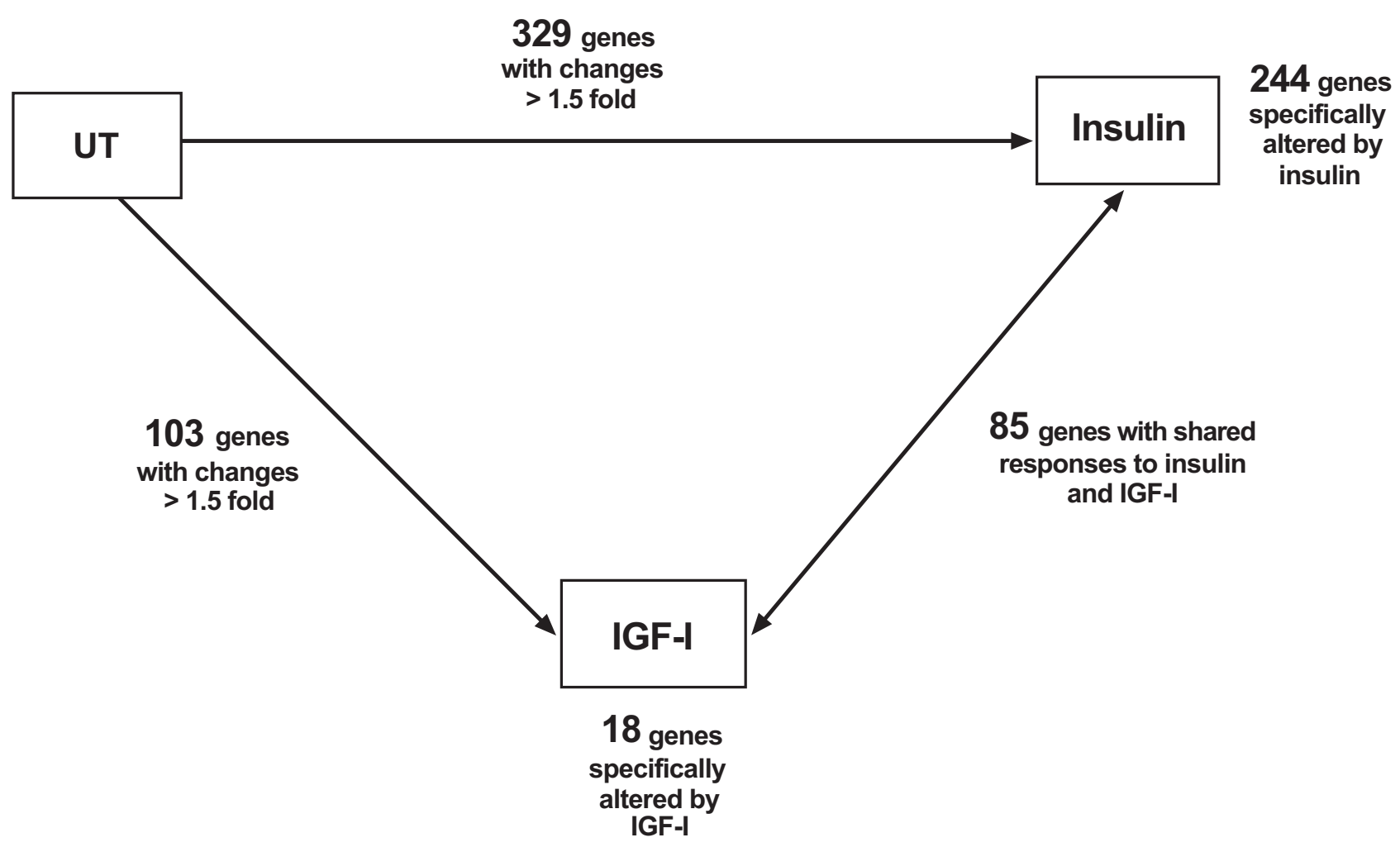

Fig. (2). Comparative anlysis of genes differentially regulated by insulin and IGF-I, perfomed in dChip using "combine type" terms like Or, And/And Not. These analyses revealed that 329 genes were regulated by insulin and 103 by IGF-I, from these 244 were specifically regulated by insulin and 18 specifically by IGF-I, whereas 85 genes were shared by both ligands.

Before uploading, we split our data set into up- and down-regulated genes in both treatments and then ran a comparison analysis. From this several other assignments to the ontological categories were found possible. As shown in Tables $\mathbf{1}$ and 2, IPA assigned the largest number of up- and down-regulated transcripts to the categories of lipid metabolism, cell death/apoptosis, cell cycle, cell growth and proliferation, cell signaling, cell morphology, post translational modification, organ development, embryonic development, and carbohydrate metabolism.

Among the differentially regulated transcripts, we found genes that were previously reported to be regulated by insulin and IGF-I. For example, the expression of PCK1 (phosphoenolpyruvate carboxykinase 1), a gene involved in regulation of gluconeogenesis [13], was downregulated by both insulin and IGF-I. Two other genes previously reported as being differentially expressed in microarray experiments, pyruvate dehydrogenase kinase 4 (PDK4) and integrin $\alpha-6$ (ITGA6) $[5,6]$ were expressed in similar fashion, thus providing evidence for the validity of the obtained results.

\section{Similar and Differentially Regulated Intracellular Signal- ing Pathways by Insulin and IGF-I}

The key issue in understanding the specificity of signaling by insulin and insulin-like growth factors is whether they act on pathways that can use different subsets within a given signaling network (that may then translate into distinct subsets of differentially expressed genes), or act via the same pathways but with different outputs (e.g. with different kinetics of activation of some key pathway elements), which may result in quantitative rather than qualitative differences in the patterns of gene expression.

In an attempt to relate such differentially expressed genes to distinct signaling pathways, we uploaded the full data set for both treatments into the Ingenuity Pathway Analysis (IPA) software and identified signaling pathways from the IPA library of canonical pathways affected by treatments with insulin or IGF-I. IPA identified that a small set of genes involved in the phosphatidylinositol 3-kinase (PI3K/AKT) signaling pathway, was specifically up-regulated in our dataset by insulin, namely activator of heat-shock $90-\mathrm{kd}$ protein ATPase 1 (AHSA1) and stress-induced phosphoprotein 1 (STIP1), whereas heat shock protein-90 (Hsp90) was also upregulated by IGF-I. Fig. (4) displays a schematic representation for the insulin stimulated PI3K/AKT signaling pathways generated by IPA, with members of the pathway found in our data set in red.

Another pathway identified by IPA as being significant, is the extracellular signal-regulated kinase/ mitogenactivated protein kinase (ERK/MAPK) signaling pathway. Four genes which were differentially regulated by insulin in this pathway were all upregulated while 2 were up-regulated and 1 down-regulated by IGF-I. The genes associated with ERK/MAPK pathway, regulated by insulin were heat shock $27 \mathrm{kDa}$ protein 1 (HSPB1), phosphoinositide-3-kinase, regulatory subunit 3 (PIK3R3) and protein phosphatase 1, regulatory (inhibitor) subunit 3C (PPPIR3C) (Fig. 5). 
Table 1. Genes Upregulated by Insulin and IGF-I Associated with Major Biological Functions

\begin{tabular}{|c|c|c|c|c|c|}
\hline Function & Symbol & Description & Genbank ID & IGF-I & Insulin \\
\hline \multicolumn{6}{|c|}{ Lipid Metabolism } \\
\hline & HMGCR & 3-hydroxy-3-methylglutaryl-Coenzyme A reductase & AL518627 & 2.39 & 4.25 \\
\hline & FDPS & farnesyl diphosphate synthase & NM_002004.1 & 3.29 & 3.48 \\
\hline & ACLY & ATP citrate lyase & U18197.1 & & 3.96 \\
\hline & ACSL3 & acyl-CoA synthetase long-chain family member 3 & D89053.1 & & 1.99 \\
\hline & ANXA1 & annexin $\mathrm{A} 1$ & NM_000700.1 & & 1.99 \\
\hline & CYCS & cytochrome c, somatic & ВC005299.1 & & 2.59 \\
\hline & IDI1 & isopentenyl-diphosphate delta isomerase 1 & NM_004508.1 & & 2.94 \\
\hline & IL1B & interleukin 1 , beta & M15330 & & 2.02 \\
\hline & NSDHL & $\mathrm{NAD}(\mathrm{P})$ dependent steroid dehydrogenase-like & BC000245.1 & & 2.73 \\
\hline & PPP1R3C & protein phosphatase 1 , regulatory (inhibitor) subunit $3 \mathrm{C}$ & N26005 & & 2.23 \\
\hline & ST6GALNAC4 & Homo sapiens ST6 & NM_014403.1 & & 2.03 \\
\hline & STIP1 & stress-induced-phosphoprotein 1 (Hsp70/Hsp90-organizing protein) & BE886580 & & 2.84 \\
\hline \multicolumn{6}{|c|}{ Cell Death/Apoptosis } \\
\hline & CYCS & cytochrome c, somatic & ВC005299.1 & & 2.59 \\
\hline & MICA & MHC class I polypeptide-related sequence A & NM_000247.1 & & 1.8 \\
\hline & OGG1 & 8-oxoguanine DNA glycosylase & NM_016820.1 & & 3.62 \\
\hline & PIK3R & phosphoinositide-3-kinase, regulatory subunit, polypeptide 3 (p55 $\gamma$ ) & BE622627 & & 2.41 \\
\hline \multicolumn{6}{|l|}{ Cell Cycle } \\
\hline & ATR & ataxia telangiectasia and $\operatorname{Rad} 3$ related & U49844.1 & & 1.75 \\
\hline & CCND3 & cyclin D3 & NM_001760.1 & & 2.09 \\
\hline & DUSP1 & dual specificity phosphatase 1 & NM_004417.2 & & 1.73 \\
\hline & SMARCA4 & $\begin{array}{l}\text { SWI/SNF related, matrix associated, actin dependent regulator of chroma- } \\
\text { tin }\end{array}$ & D26156.1 & & 1.82 \\
\hline & TUBB & tubulin, beta polypeptide & NM001069 & 3.7 & 3.1 \\
\hline & PHB & prohibitin & NM_002634.2 & & 1.92 \\
\hline \multicolumn{6}{|c|}{ Cell Growth \& Proliferation } \\
\hline & GPR30 & G protein-coupled receptor 30 & U58828.1 & 2.46 & 3.12 \\
\hline & HMGCR & 3-hydroxy-3-methylglutaryl-Coenzyme A reductase & AL518627 & 2.39 & 4.25 \\
\hline & IGF1 & insulin-like growth factor 1 (somatomedin C) & NM_000618.1 & 2.53 & \\
\hline & PFN1 & profilin 1 & NM_005022.1 & 2.27 & 1.79 \\
\hline & PKM2 & pyruvate kinase, muscle & NM_002654.1 & 3.09 & \\
\hline
\end{tabular}




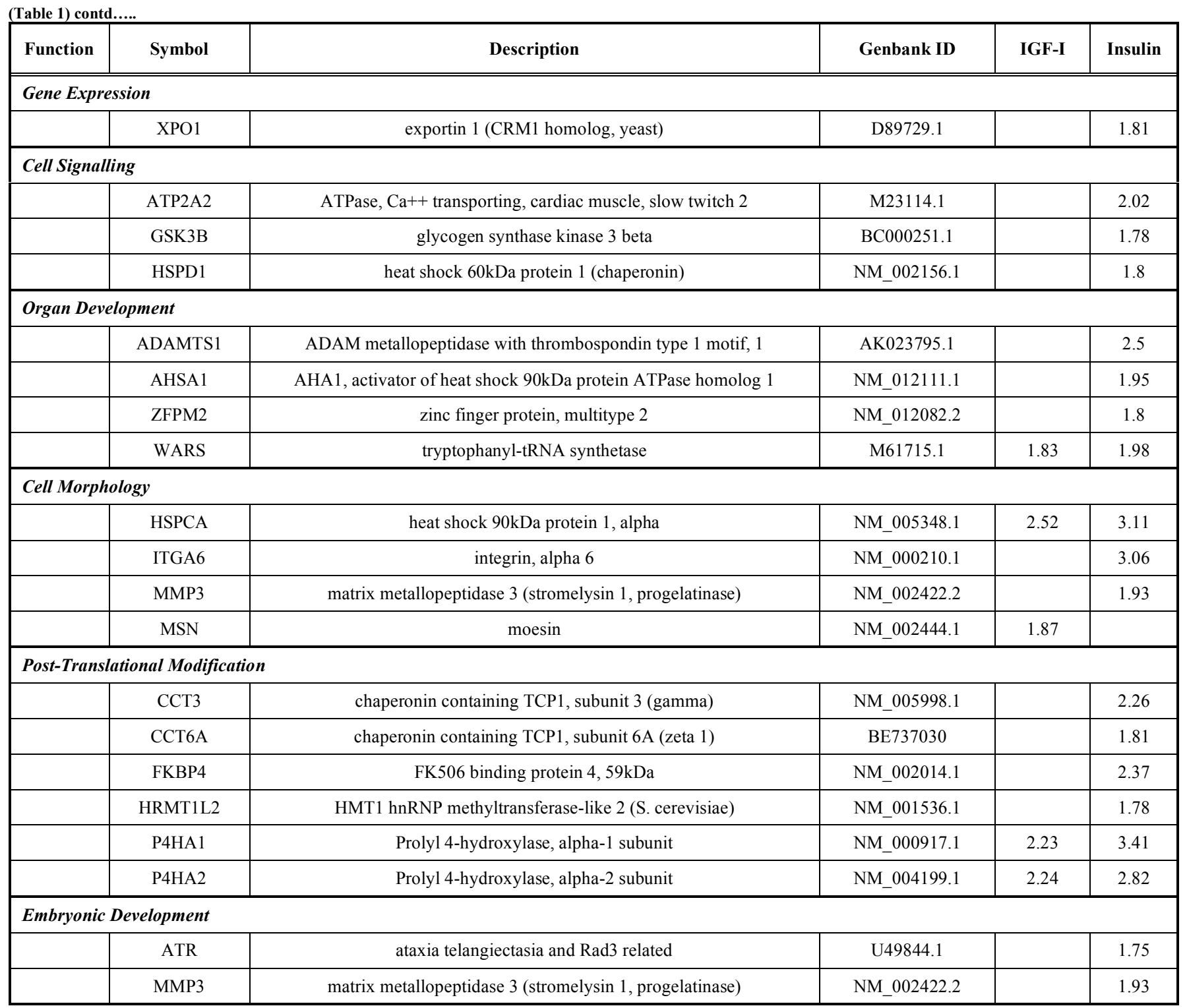

From the list of genes reported by dChip to be significant, in both treatments we split the obtained lists in up and dowregulated genes and uploaded into the IPA application. The functional analysis identified the biological functions that were most significant to the data set uploaded. Not all genes could be associated with the identified biologcal functions, whereas others were found in multiple categories. Values represent fold changes in expression.

Other interesting genes identified by this microarray study include the adipokine, visfatin (VF), or Pre-B-Cell Colony-Enhancing Factor 1 (PBEF1), and Sirtuin 1 (Sirt 1), Growth Hormone Receptor (GHR), farnesyl diphosphate synthase (FTPS) and tubulin, beta polypeptide (TUBB/BT2) (Tables 1, 2 and Fig. (6)).

\section{Validation of Microarray Data}

To validate microarray gene expression data, 7 genes belonging to different classes of categories were selected for real-time PCR analysis. In addition to specific primers for each gene, we used short hydrolysis probes of 8-9 nucleotides, thereby increasing the specificity. The selected genes, sequences of primer pairs and the Universal Library Probe numbers used for the real-time PCR are reported in (Table 3). The results obtained by real-time PCR were compared with microarray data to check for the robustness of the microarray experiments.
The levels of the seven genes validated by real-time PCR were generally higher than the levels obtained by microarray analysis, but otherwise followed the general expression patterns. The expression profiles by the two techniques are shown in (Fig. 6). The IGF-I transcript did not show up in the microarray analysis in the insulin treatment but was 2-fold upregulated in real-time PCR analysis. Generating a complete list of all genes with fold-regulation and p-values, revealed though, that IGF-I was actually also upregulated 1.6-fold in the microarray analysis but the p-value was higher than the cutoff we had chosen and was therefore not found significant by the analysis performed by dChip. Moreover, the transcript, Sirtuin, identified as downregulated by PCR method was also found in the IGF-1 list but the fold regulation was -1.4, while we had set the lower boundary fold change to \pm 1.5 and therefore was not initially detected by microarray. In summary, these results show that it is important to verify the microarray results by using other techniques. 
Table 2. Genes Downregulated by Insulin and IGF-I Associated with Major Biological Functions

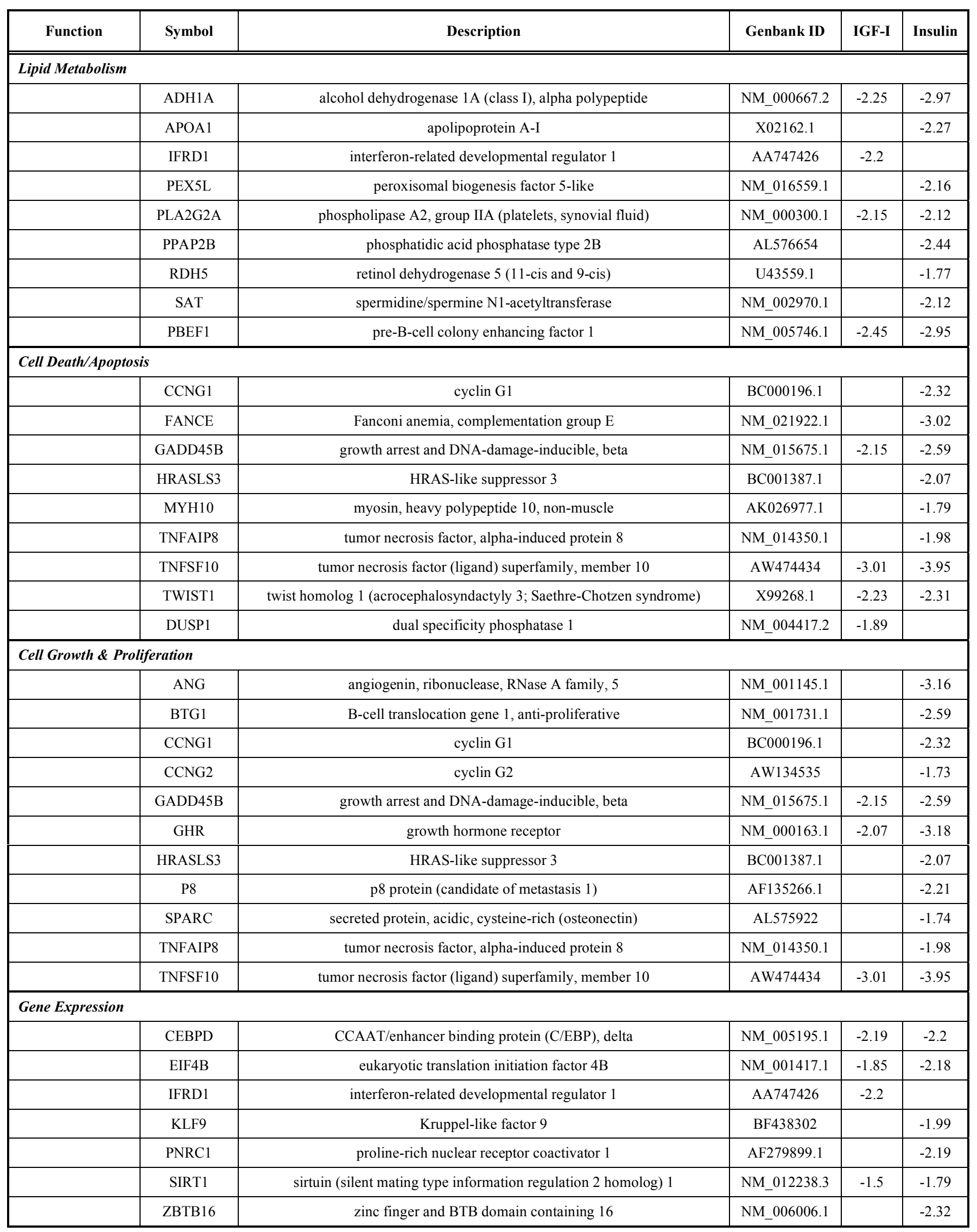




\begin{tabular}{|c|c|c|c|c|c|}
\hline Function & Symbol & Description & Genbank ID & IGF-I & Insulin \\
\hline \multicolumn{6}{|l|}{ Cell Cycle } \\
\hline & CDKN1B & cyclin-dependent kinase inhibitor 1B (p27, Kip1) & ВC001971.1 & & -2.12 \\
\hline & CDKN1C & cyclin-dependent kinase inhibitor 1C (p57, Kip2) & R78668 & & -2.3 \\
\hline & DTNA & dystrobrevin, alpha & U26744.1 & & -1.96 \\
\hline & EIF4B & eukaryotic translation initiation factor 4B & NM_001417.1 & -1.85 & -2.18 \\
\hline & GHR & growth hormone receptor & NM_000163.1 & -2.07 & -3.18 \\
\hline & HBP1 & HMG-box transcription factor 1 & AF019214.1 & & -2.58 \\
\hline & MAOA & monoamine oxidase $\mathrm{A}$ & NM_000240.1 & & -2 \\
\hline & PEX1 & peroxisome biogenesis factor 1 & NM_000466.1 & & -1.87 \\
\hline & RBL2 & retinoblastoma-like $2(\mathrm{p} 130)$ & NM_005611.1 & -1.92 & -2.79 \\
\hline & TNFSF10 & tumor necrosis factor (ligand) superfamily, member 10 & AW474434 & -3.01 & -3.95 \\
\hline & ZBTB16 & zinc finger and BTB domain containing 16 & NM_006006.1 & & -2.32 \\
\hline \multicolumn{6}{|c|}{ Carbohydrate Metabolism } \\
\hline & GYG2 & glycogenin 2 & U94357.1 & & -1.99 \\
\hline & PCK1 & phosphoenolpyruvate carboxykinase 1 (soluble) & NM_002591.1 & -3.61 & -4.51 \\
\hline & PDK4 & pyruvate dehydrogenase kinase, isozyme 4 & NM_002612.1 & & -2.51 \\
\hline \multicolumn{6}{|c|}{ Cell Morphology } \\
\hline & DTNA & dystrobrevin, alpha & $\mathrm{U} 26744.1$ & & -1.96 \\
\hline & GHR & growth hormone receptor & NM_000163.1 & -2.07 & -3.18 \\
\hline & MYH10 & myosin, heavy polypeptide 10 , non-muscle & AK026977.1 & & -1.79 \\
\hline & PDE3B & phosphodiesterase 3B, cGMP-inhibited & AA 888858 & -2.15 & -2.51 \\
\hline & PKP2 & plakophilin 2 & NM_004572.1 & -2.17 & -3.76 \\
\hline
\end{tabular}

\section{DISCUSSION}

In the present work, we chose to gain insights into gene expression patterns differentially regulated by insulin and IGF-I, using human adipocytes as our model, as they represent a major target for both insulin and IGF-I mediated responses in humans. We chose to use the SGBS cell line derived from the adipose tissue of a child with SimpsonGolabi-Behmel syndrome (SGBS). This spontaneously transformed cell line can undergo differentiation into adipocytes, and the cells are morphologically, biochemically and functionally identical to in vitro differentiated adipocytes from healthy subjects, and in our opinion represent a better physiological model for studying IGF-I and insulin signaling in humans, rather than NIH-3T3 differentiated cells which are mouse derived $[9,10]$.

Initially, we wished to study gene expression profiles for non differentiated preadipocytes as well as adipocytes but the SGBS preadipocytes showed very poor responsiveness to either ligand and were therefore excluded from the present study (data not shown). This is particularly intriguing as both insulin and IGF-I have been shown to induce differentiation of 3T3-F442A cells into adipocytes [14]. In our hands, differentiated adipocytes responded well to both ligands, and showed greater responses to the effects of insulin (329 genes) than to IGF-I (103 genes) respectively (Fig. 2). Of these, 215 transcripts were up-regulated by insulin while 114 were down-regulated, while 70 transcripts were up-regulated and 34 down-regulated by IGF-I. This is in contrast to the earlier data reported by Dupont et al. [5] and Mulligan et al. who found greater responses to IGF-I than insulin, albeit using different cell lines. The greater response to IGF-I signaling, reported by these two groups, could be due to the cell lines used (NIH3T3 or NIH3T3-L1) and may be a consequence of the different amounts of IR and IGF-IR present in these cells. Differentiated NIH-3T3-L1 adipocytes express very high levels of IR (approximately $250 \times 10^{3}$ per cell) [15], and IGF-IR (approximately $13 \times 10^{3}$ receptors per cell) [16], while NIH-3T3 express low levels of IR $(5.4 \pm 1.35 \mathrm{x}$ $\left.10^{3}\right)$ and IGF-IR $\left(3.4 \pm 2.6 \times 10^{3}\right)$ [17]. While the levels of IR and IGF-1R in SGBS adipocytes are unknown, both receptors are known to be expressed in these cells [10], and further emphasizes the potential for marked differences in responses to insulin or IGF-I signaling between murine and human adipocytes.

In agreement with Mulligan et al. [6] and in contrast to Dupont et al. [5] we found more similarly regulated genes than differentially regulated by the two hormones in microarray analysis. However, since insulin and IGF-I target similar intracellular signaling pathways and have generally overlapping effects in tissue culture; one might expect more similarities than differences in gene expression patterns between 
the two ligands, though some differences also exist, as the ERK/MAPK pathway was specifically activated by IGF-I in our study. In this regard, our results in differentiated SGBS adipocytes are also in agreement with those observed by us in insulin receptor transfected L6 myoblasts [7]. Microarray analysis of gene expression changes in these cells when stimulated by insulin found that half of the differentially regulated genes interacted in some way with genes associated with the MAPK (mitogen-activated protein kinase) pathway [8]. These results are also in contrast to the observations we have made in human myoblasts or myotubes, where IGF-I was found to be the most potent regulator of gene expression [7].

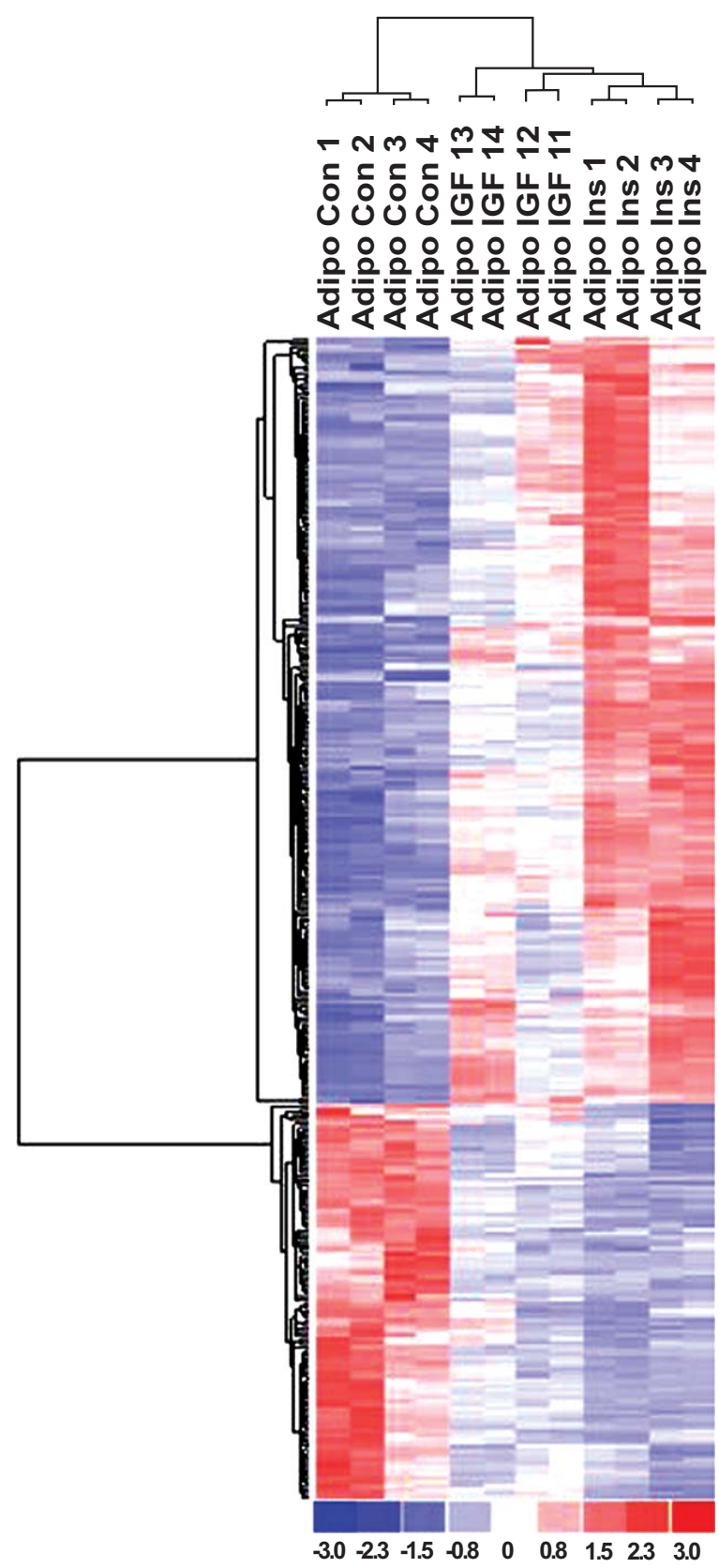

Fig. (3). Hierarchical cluster analysis showing gene expression changes in SGBS adipocytes (including redundant probe sets and genes of unknown functions). Greatest responses were observed for insulin. Increases are shown in red while decreases in expression levels are shown in blue. Genes and samples are clustered.
PCK1 gene involved in gluconeogenesis was downregulated by insulin and IGF-I in agreement with previously published data for brown adipocytes [13], and white adipose tissue [18]. PDK4, which is involved in glucose and lipid metabolism [19] was down-regulated specifically by insulin but not by IGF-I in our data, whereas in contrast to our results Mulligan et al. found it down-regulated by both ligands [6]. Our results (i.e. down-regulation of PDK4 by insulin) fit with PDK4's role in inactivating pyruvate dehydrogenase, and promoting fatty acid oxidation. In vivo, secretion of insulin responds to high levels of carbohydrates and would therefore downregulate PDK4, thereby promoting the oxidation of glucose instead of fatty acids [20].

Next we used IPA software to identify key biological pathways associated with the genes identified by microarray analysis. Two major RTK signaling pathways were identified, PI3K/AKT (Fig. 4) and ERK/MAPK (Fig. 5).

To confirm the microarray results we validated the differential expression of seven genes, selected from different classes of categories by real-time PCR analysis. Although the numbers of genes selected for validation by PCR are small, the results indicate the robustness of the microarray analysis. Of these visfatin (also known as Pre-B-Cell Colony-Enhancing Factor 1; PBEF1 or Nicotinamide Phophoribosyltransferase; NAMPT) was found by us to be a gene whose expression is regulated by insulin and IGF-I (Table 2, Fig. 6). We also investigated its expression in untreated adipocytes compared to untreated preadipocytes and found that it is upregulated in adipocytes, and downregulated when the adipocytes are stimulated by insulin or IGF-I as confirmed by real-time PCR (data not shown). Visfatin is especially interesting because, visfatin has been shown to have a marked increase during the differentiation of NIH-3T3-L1 preadipocytes, and is downregulated in adipocytes in response to insulin treatment [21]. Elevated levels of visfatin have been observed patients with type 2 diabetes compared to normal subjects [22]. Interestingly, we observed that the expression of the adipokine visfatin was down-regulated by both insulin and IGF-I in the differentiated adipocytes. When the levels of non-stimulated adipocytes were compared with non-stimulated pre-adipocytes, we found it to be more than 6 -fold higher in adipocytes. Furthermore, Chen et al. measured the plasma level of visfatin in patients with T2DM and found it to be 2-fold higher than in control subjects [22], suggesting a role in pathogenesis of T2DM. Thus, the fact that we also found the expression of visfatin upregulated in untreated adipocytes (controls) and downregulated when treated with insulin, further enhances its role in adipogenesis and diabetes.

Another gene found by us to be affected by insulin or IGF-I was Beta-tubulin. In our system, this gene was upregulated by either insulin or IGF-I (Table 1, Fig. 6). This is in contrast to data from rat primary adipocytes where protein levels of beta-tubulin decrease in response to treatment with insulin [23]. The functional significance of this may relate to tubulin's roles in regulating Glut4 translocation in adipocytes and other insulin sensitive cells [24]. Farnesyl diphosphate synthase (FDPS) also known as Farnesylpyrophosphate synthase (FPS) was found by us to also be upregulated by insulin and IGF-I (Table 2, Fig. 6). In hepatocytes, insulin 


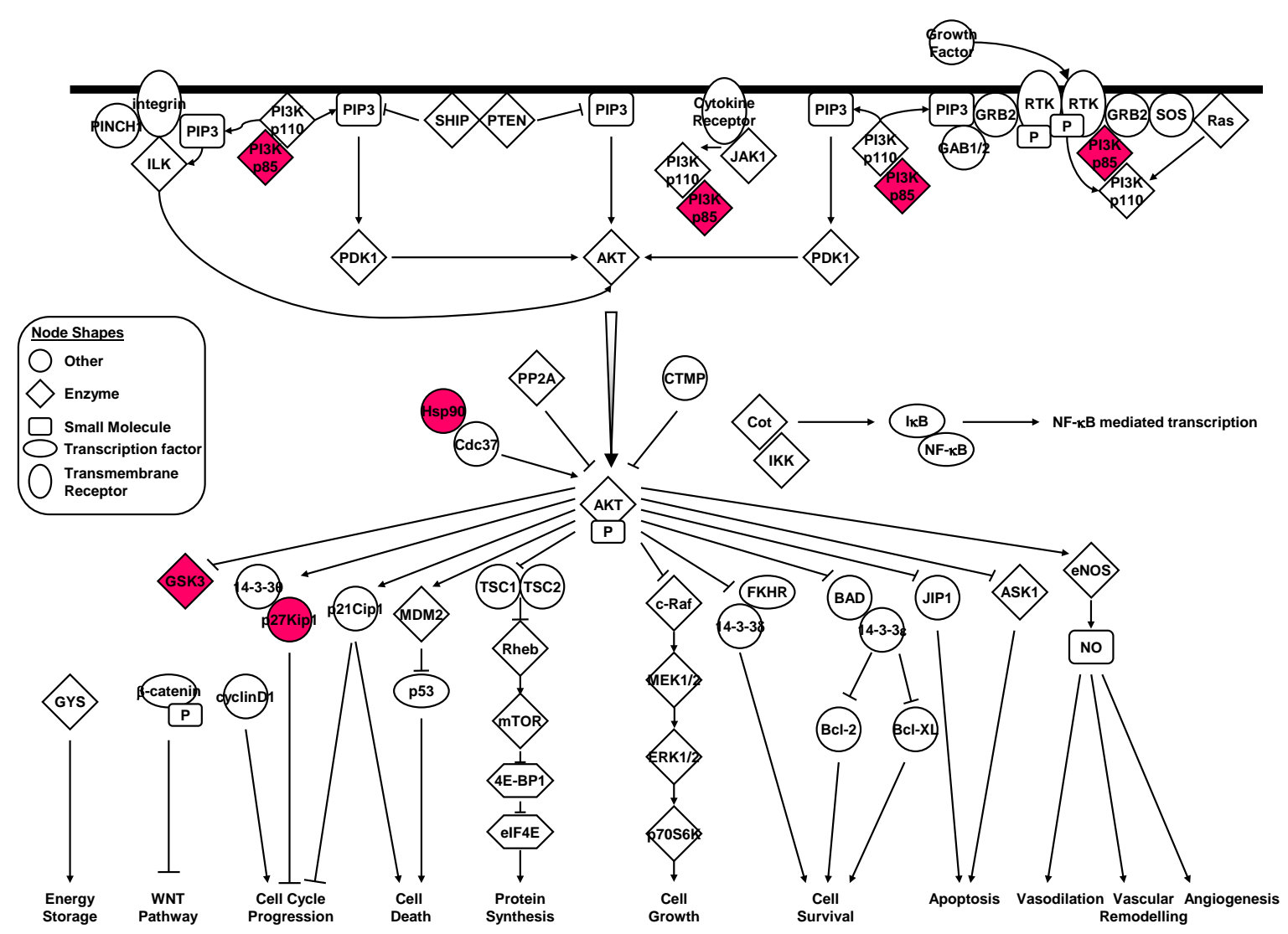

Fig. (4). Graphic representation of insulin regulated PI3K/AKT pathway generated by IPA. HSP90, PI3K, GSK3 and p27kip1 (CDKN1B) displayed in red, represent genes found in the data set differentially regulated by insulin. All except CDKN1B were upregulated.

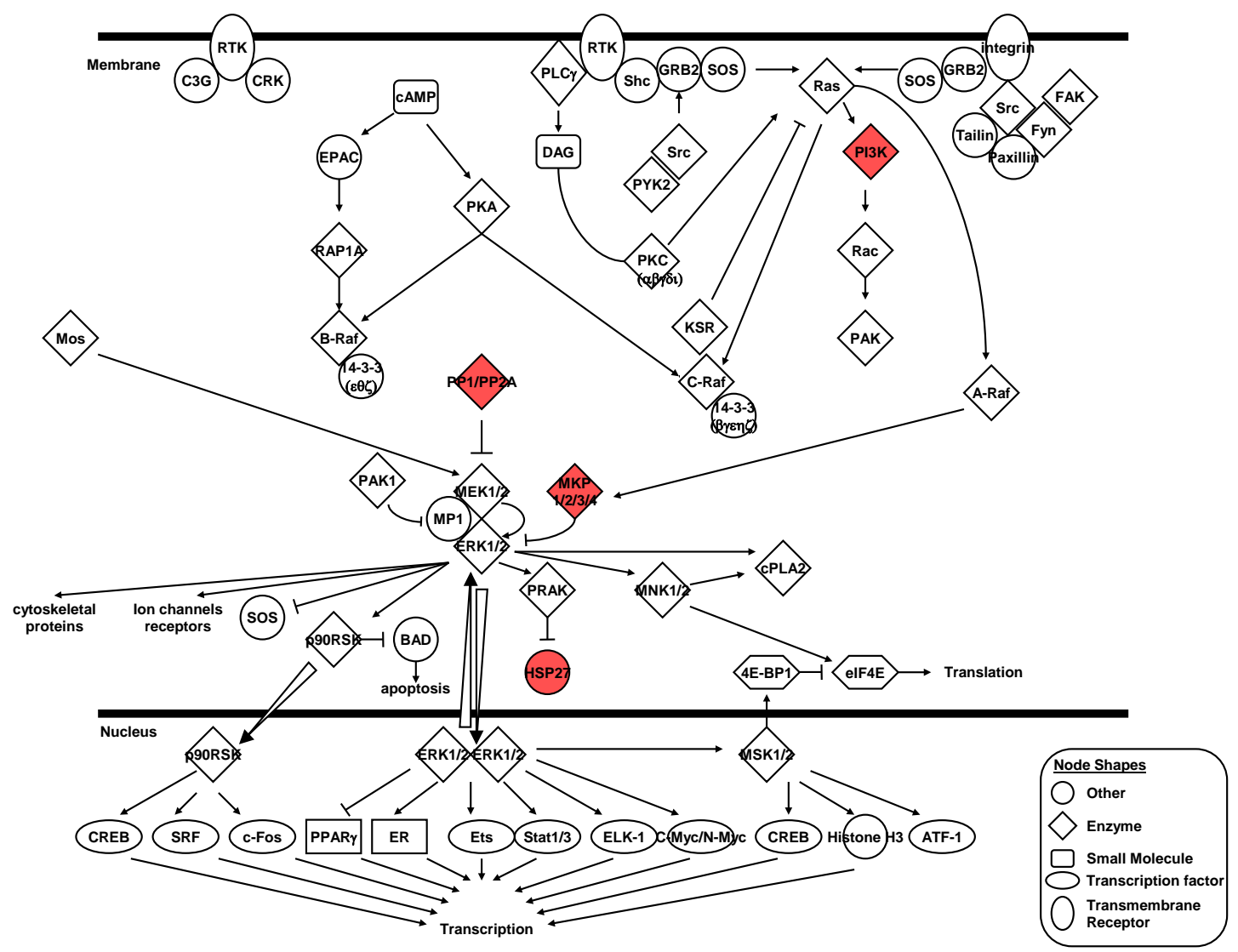

Fig. (5). Graphic summary of insulin regulated ERK/MAPK pathway generated by IPA. The genes in red colour, MKP1/2/3/4 (DUSP1), PP1/PP2A (PPP1R3C) and Hsp27 (HSPB19), representing genes found in the uploaded dataset, were all upregulated by insulin. 


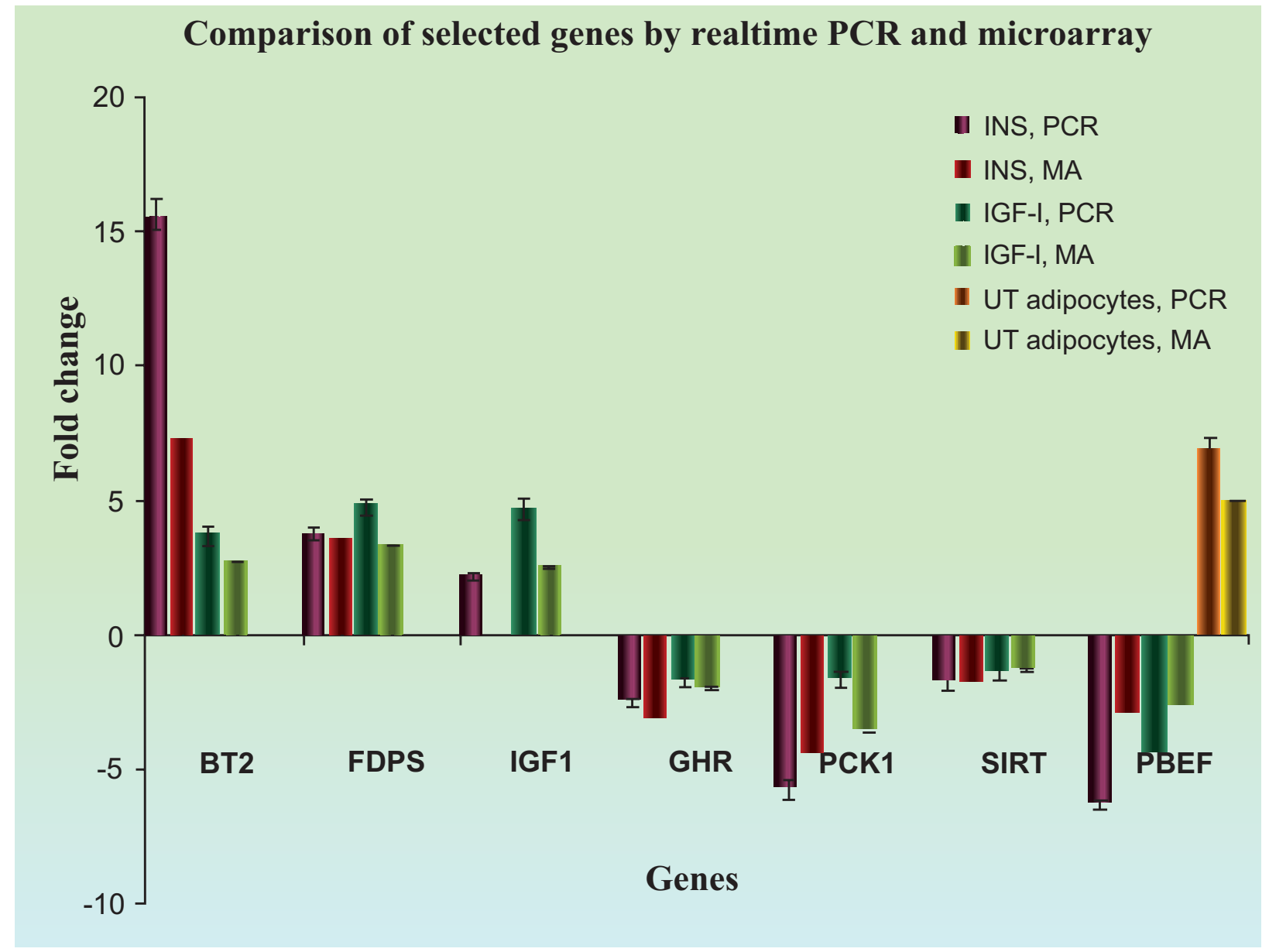

Fig. (6). Comparison of patterns in genes differentially regulated by insulin and IGF-I. The expression profile of seven selected genes were validated by real-time PCR (PCR) and compared with the expression profile found by microarray (MA). The expression of PBEF was also compared in untreated adipocytes with untreated preadipocytes. The fold induction of the real-time PCR data was computed using a standard curve analysis and normalized to the level of $18 \mathrm{~S}$ rRNA. At least two independent experiments were performed in duplicates for each gene.

Table 3. Primer Sequences and Universal Probe Library (Roche) Probe Numbers Used for Real-Time PCR

\begin{tabular}{|c|c|c|}
\hline Gene & UPL Probe & Primer Sequence \\
\hline Beta Tubulin 2 & 70 & $\begin{array}{l}\text { ttgtctacttcctcctgcttcc (left) } \\
\text { ctgatcacctcccaaaacttg (right) }\end{array}$ \\
\hline Farnesyl diphosphate synthase & 75 & $\begin{array}{l}\text { gccactccagaacagtaccag (left) } \\
\text { cctcatatagcgccttcacc (right) }\end{array}$ \\
\hline Growth hormone receptor & 59 & $\begin{array}{l}\text { cgttcacctgagcgagaga (left) } \\
\text { tgagtccattcttgagtgttcc (right }\end{array}$ \\
\hline insulin-like growth factor 1 & 75 & $\begin{array}{l}\text { tggagacaggggcttttattt (left) } \\
\text { aggggtgcgcaatacatc (right) }\end{array}$ \\
\hline Visfatin & 65 & $\begin{array}{c}\text { gccagcagggaattttgtta (left) } \\
\text { gccattcttgaagacagtatgga (right) }\end{array}$ \\
\hline phosphoenolpyruvate carboxykinase 1 & 75 & $\begin{array}{l}\text { caacctggccatgatgaac (left) } \\
\text { tgatggcccttaaatgacct (right) }\end{array}$ \\
\hline sirtuin & 70 & $\begin{array}{l}\text { tgtacgacgaagacgacgac (left) } \\
\text { ttcatcaccgaacagaaggtt (right) }\end{array}$ \\
\hline 18S rRNA & 77 & $\begin{array}{l}\text { ctcaacacgggaaacctcac } \\
\text { cgctccaccaactaagaacg }\end{array}$ \\
\hline
\end{tabular}


promotes lipogenesis, while glucagon inhibits this. During fasting the expression levels of central lipogenic genes, including farnesyl pyrophosphate synthase, are decreased in primary hepatocytes by glucagon but increased by the inhibition of p38 MAPK [25]. In a manner analagous to that obbserved for hepatic lipogenesis, as insulin signals through the MAPK pathway, FPS may therefore be a good candidate target gene regulated via insulin-MAPK signalling, and may therefore play an important role in adipogenesis.

Genes which were downregulated by insulin and IGF-I in adipocytes include the growth hormone recptor (GHR) and sirtuin 1 (Sirt1). It is well established that the GHR plays important roles in the signal transduction pathway leading to synthesis and secretion of insulin-like growth factor I [26]. Expression of GHR has recently been observed to increase during adipocyte differentiation and to maintain high levels of expression in mature adipocytes [27, 28]. Intriguingly, $\mathrm{GH}$ is known to show mitogenic activity in preadipocytes by stimulating IGF-I expression up to 12 -fold [29, 30], but in differentiated adipocytes GH was shown to elicit antiadipogenic activity [29]. Insulin or IGF-I signaling in adipocytes may therefore function to regulate the levels of expression of this receptor, as overexpression of this gene may be deleterious to adipocytes by inhibiting glucose uptake and lipogenesis, and stimulating lipolysis [29, 31]. Sirt1 has been implicated as an important regulator of insulin and IGF-I mediated signaling $[32,33]$. Indeed SIRT1 is downregulated in insulin-resistant cells and tissues and knockdown or inhibition of SIRT1 induces insulin resistance [34]. Why insulin or IGF-I signaling results in decreased gene expression of SIRT1 in our hands is not clear, but such a decrease may reflect a transient response by the cells to these signaling events.

One of the major problems associated with microarray studies concerns the frequent lack of overlap in identified genes from similar studies. Several factors may therefore have confounded the results of this analysis. For instance, SGBS adipocytes express both IR and IGF-IR [10], and therefore there remains the possibility that ligand crossreactivity occurred as well as the formation of hybrid receptors which may play dilute or cause divergent gene expression. Another factor to consider is the timescale chosen for stimulation. We chose to stimulate for $24 \mathrm{~h}$ in order to get maximal responses but this will also trigger some secondary gene expression events, while early and immediate early gene expression events may be missed. Previously similar gene expression studies have used $90 \mathrm{~min}$ [5] and $4 \mathrm{~h}$ [6], which would miss the later gene expression events. The ideal study would include several time points as well as several concentrations of the ligands, and future experiements should consider these. Nevertheless, our data compare quite well with the previous reported microarray results $[5,6]$. As discussed these differences may arise from the use of different model system, different time points and concentrations of the ligands. It may prove useful to do a combined metaanalysis of all the available microarray data from similar studies to identify some common patterns, which might reveal some interesting and yet not discovered expression profiles and pathways related to insulin or IGF-I signaling.

\section{CONCLUSION}

Our results indicate that under the conditions used in the present study, insulin is a more potent regulator of gene expression in human adipocytes than IGF-I.

\section{ACKNOWLEDGEMENTS}

We thank Prof. M. Wabitsch for providing the SGBS preadipocytes. We also thank the Microarray Center at Rigshospitalet (Copenhagen University Hospital) and Susanne Smed for assistance in scanning the Affymetrix microarrays. Birgit Klaproth is acknowledged for technical assistance. Tina Olesen is acknowledged for taking care of SGBS cell culture and extracting the total RNA. This work was partly supported by a PhD fellowship from the Medicon Valley Academy to Shaukat Mahmood. Steven G. Gray was supported by a postdoctoral fellowship from the BIO+IT program of the Øresund IT Academy (Denmark). The Receptor Systems Biology Laboratory and the Hagedorn Reseach Institute are independent basic research components of Novo Nordisk A/S.

\begin{tabular}{|c|c|c|}
\hline \multicolumn{3}{|c|}{ ABBREVIATIONS } \\
\hline BT2 & $=$ & Beta tubulin 2 \\
\hline FDPS & $=$ & Farnesyl di-phosphate synthase \\
\hline IGF1 & $=$ & Insulin-like growth factor 1 \\
\hline GHR & $=$ & Growth hormone receptor \\
\hline PCK1 & $=$ & Phosphoenolpyruvate carboxykinase 1 \\
\hline SIRT1 & $=$ & $\begin{array}{l}\text { Sirtuin, silent mating type information } \\
\text { regulation } 2 \text { homolog }\end{array}$ \\
\hline PBEF & $=$ & $\begin{array}{l}\text { pr-B-cell colony-enhancing factor } 1, \\
\text { visfatin }\end{array}$ \\
\hline UT & $=$ & Untreated \\
\hline MA & $=$ & Microarray \\
\hline
\end{tabular}

\section{REFERENCES}

[1] Werner H, Weinstein D, Bentov I. Similarities and differences between insulin and IGF-I: structures, receptors, and signalling pathways. Arch Physiol Biochem 2008; 114: 17-22.

[2] De Meyts P. The insulin receptor: a prototype for dimeric, allosteric membrane receptors? Trends Biochem Sci 2008; 33: 376-84.

[3] De Meyts P, Palsgaard J, Sajid W, Theede AM, Aladdin H. Structural biology of insulin and IGF-1 receptors. Novartis Found Symp 2004; 262: 160-71; discussion 71-6, 265-8.

[4] Leroith D, Accili D. Mechanisms of disease: using genetically altered mice to study concepts of type 2 diabetes. Nat Clin Pract Endocrinol Metab 2008; 4: 164-72.

[5] Dupont J, Khan J, Qu BH, Metzler P, Helman L, LeRoith D. Insulin and IGF-1 induce different patterns of gene expression in mouse fibroblast NIH-3T3 cells: identification by cDNA microarray analysis. Endocrinology 2001; 142: 4969-75.

[6] Mulligan C, Rochford J, Denyer G, et al. Microarray analysis of insulin and insulin-like growth factor-1 (IGF-1) receptor signalling reveals the selective up-regulation of the mitogen heparin-binding EGF-like growth factor by IGF-1. J Biol Chem 2002; 277: 424807.

[7] Palsgaard J, Brown AE, Jensen M, Borup R, Walker M, De Meyts P. Insulin-like growth factor I (IGF-I) is a more potent regulator of gene expression than insulin in primary human myoblasts and myotubes. Growth Horm IGF Res 2009; 19: 168-78. 
[8] Jensen M, Palsgaard J, Borup R, de Meyts P, Schaffer L. Activation of the insulin receptor (IR) by insulin and a synthetic peptide has different effects on gene expression in IR-transfected L6 myoblasts. Biochem J 2008; 412: 435-45.

[9] Wabitsch M, Brenner RE, Melzner I, et al. Characterization of a human preadipocyte cell strain with high capacity for adipose differentiation. Int J Obes Relat Metab Disord 2001; 25: 8-15.

[10] Fischer-Posovszky P, Newell FS, Wabitsch M, Tornqvist HE. Human SGBS cells - a unique tool for studies of human fat cell biology. Obesity Facts 2008; 1: 184-9.

[11] Li C, Wong WH. Model-based analysis of oligonucleotide arrays: expression index computation and outlier detection. Proc Natl Acad Sci USA 2001; 98: 31-6.

[12] Fischer P, Moller P, Bindl L, et al. Induction of adipocyte differentiation by a thiazolidinedione in cultured, subepidermal, fibroblastlike cells of an infant with congenital generalized lipodystrophy. $\mathrm{J}$ Clin Endocrinol Metab 2002; 87: 2384-90.

[13] Teruel T, Valverde AM, Benito M, Lorenzo M. Insulin-like growth factor I and insulin induce adipogenic-related gene expression in fetal brown adipocyte primary cultures. Biochem J 1996; 319: 62732.

[14] Christoffersen CT, Tornqvist H, Vlahos CJ, et al. Insulin and insulin-like growth factor-I receptor mediated differentiation of 3T3F442A cells into adipocytes: effect of PI 3-kinase inhibition. Biochem Biophys Res Commun 1998; 246: 426-30.

[15] Rosen OM, Chia GH, Fung C, Rubin CS. Tunicamycin-mediated depletion of insulin receptors in 3T3-L1 adipocytes. J Cell Physiol 1979; 99: 37-42.

[16] Smith PJ, Wise LS, Berkowitz R, Wan C, Rubin CS. Insulin-like growth factor-I is an essential regulator of the differentiation of 3T3-L1 adipocytes. J Biol Chem 1988; 263: 9402-8.

[17] Lammers R, Gray A, Schlessinger J, Ullrich A. Differential signalling potential of insulin- and IGF-1-receptor cytoplasmic domains. EMBO J 1989; 8: 1369-75.

[18] Foufelle F, Gouhot B, Perdereau D, Girard J, Ferre P. Regulation of lipogenic enzyme and phosphoenolpyruvate carboxykinase gene expression in cultured white adipose tissue. Glucose and insulin effects are antagonized by cAMP. Eur J Biochem 1994; 223: 893900.

[19] Kwon HS, Harris RA. Mechanisms responsible for regulation of pyruvate dehydrogenase kinase 4 gene expression. Adv Enzyme Regul 2004; 44: 109-21.

[20] Sugden MC, Holness MJ. Mechanisms underlying regulation of the expression and activities of the mammalian pyruvate dehydrogenase kinases. Arch Physiol Biochem 2006; 112: 139-49.
[21] MacLaren R, Cui W, Cianflone K. Visfatin expression is hormonally regulated by metabolic and sex hormones in 3T3-L1 preadipocytes and adipocytes. Diabetes Obes Metab 2007; 9: 490-7.

[22] Chen MP, Chung FM, Chang DM, et al. Elevated plasma level of visfatin/pre-B cell colony-enhancing factor in patients with type 2 diabetes mellitus. J Clin Endocrinol Metab 2006; 91: 295-9.

[23] Liu LB, Omata W, Kojima I, Shibata H. Insulin recruits GLUT4 from distinct compartments via distinct traffic pathways with differential microtubule dependence in rat adipocytes. J Biol Chem 2003; 278: 30157-69.

[24] Olson AL, Trumbly AR, Gibson GV. Insulin-mediated GLUT4 translocation is dependent on the microtubule network. J Biol Chem 2001; 27: 10706-14.

[25] Xiong Y, Collins QF, An J, et al. p38 mitogen-activated protein kinase plays an inhibitory role in hepatic lipogenesis. J Biol Chem 2007; 282: 4975-82.

[26] Rosenbloom AL. IGF-I deficiency due to GH receptor deficiency. Horm Metab Res 1999; 31: 161-71.

[27] Bäck K, Arnqvist HJ. Changes in insulin and IGF-I receptor expression during differentiation of human preadipocytes. Growth Horm IGF Res 2009; 19: 101-11.

[28] Wei Y, Rhani Z, Goodyer CG. Characterization of growth hormone receptor messenger ribonucleic acid variants in human adipocytes. J Clin Endocrinol Metab 2006; 91: 1901-8.

[29] Wabitsch M, Braun S, Hauner H, et al. Mitogenic and antiadipogenic properties of human growth hormone in differentiating human adipocyte precursor cells in primary culture. Pediatr Res 1996; 40: 450-6.

[30] Wabitsch M, Heinze E, Hauner $\mathrm{H}$, et al. Biological effects of human growth hormone in rat adipocyte precursor cells and newly differentiated adipocytes in primary culture. Metabolism 1996; 45: 34-42.

[31] Bick T, Frick GP, Leonard D, Leonard JL, Goodman HM. Overexpression of the short form of the growth hormone receptor in 3T3L1 mouse preadipocytes. Proc Soc Exp Biol Med 1994; 206: 185-9.

[32] Li Y, Xu W, McBurney MW, Longo VD. SirT1 inhibition reduces IGF-I/IRS-2/Ras/ERK1/2 signalling and protects neurons. Cell Metab 2008; 8: 38-48.

[33] Zhang J. The direct involvement of SirT1 in insulin-induced insulin receptor substrate-2 tyrosine phosphorylation. J Biol Chem 2007; 282: 34356-64.

[34] Sun C, Zhang F, Ge X, et al. SIRT1 improves insulin sensitivity under insulin-resistant conditions by repressing PTP1B. Cell Metab 2007; 6: 307-19. 